\title{
High-magnification observation of seminiferous tubules through the tunica albuginea by two-photon laser scanning microscopy
}

\author{
Vincent Achard $^{1,2}$, Pascal Weber ${ }^{3}$, Georges Mercier ${ }^{1}$ and Marie-Pierre Blanchard ${ }^{4}$ \\ Testicular sperm extraction is widely used in the treatment of male infertility in cases of non-obstructive azoospermia. Identifying \\ spermatogenetic foci within the testes is critical for testicular sperm extraction. Two-photon laser scanning microscopy (TPLSM) is an \\ autofluorescence-based microscopy technique that allows observation at a cellular level in the depth of fresh living tissues and does not \\ require any histological processing (fixation or staining). The wavelengths previously used have shown no phototoxicity on sperm. We \\ used TPLSM to detect spermatogenetic foci in fresh mouse testicular parenchyma without disrupting the tunica albuginea. Fresh \\ surgically retrieved testes were observed using TPLSM within $1 \mathrm{~h}$ after extraction. Contralateral testes for each animal were observed \\ using standard histology. Using TPLSM we were able to observe and measure the diameter of seminiferous tubules through the tunica \\ albuginea, similar to the histological control. Structures within epithelial tubules were also observed, although their nature has yet to be \\ identified. TPLSM is a real-time microscopy technique that could detect spermatogenetic foci.
}

Asian Journal of Andrology (2011) 13, 774-776; doi:10.1038/aja.2011.6; published online 11 April 2011

Keywords: seminiferous epithelium; spermatogenesis

\section{INTRODUCTION}

In non-obstructive azoospermia, testicular sperm extraction combined with intracytoplasmic sperm injection has proven to be an acceptable line of treatment. ${ }^{1}$ However, testicular sperm extraction is a blind procedure that does not identify the focal sperm-producing areas of the testicle until after tissue has been excised from the patient which implies the disruption of the testis inner connective capsule (the tunica albuginea) to allow access to the parenchyma. Preventing this disruption could limit the risk of immediate vascular damage, and secondary complications. ${ }^{2}$ Detecting spermatogenesis through the tunica albuginea during surgical time using a microscopy technique would increase sperm retrieval.

Two-photon laser scanning microscopy (TPLSM) is based on an excitation by two photons emitted at a very low time interval, which causes a higher energy electronic transition in molecules. ${ }^{3}$ Therefore, it is a high-resolution laser scanning microscopy technique that allows for deep imaging inside biological tissues. Because it reveals autofluorescent molecules within the tissue (riboflavin, $\mathrm{NADH}$, folic acid, etc.), ${ }^{4}$ it is possible to observe structures in fresh tissues without histological preparation or staining of the tissue. The ability of two-photon-based microscopy techniques to explore tissues at a cellular level, along with their low photoxicity related to the long wavelengths used, ${ }^{5,6}$ makes it a promising tool for spermatogenetic foci detection. Our objective was therefore to use TPLSM to observe testicular parenchyma through the tunica albuginea on freshly retrieved testes in mice without any drug administration to the animal or tissue processing prior to observation.

\section{MATERIALS AND METHODS}

Animal experiments were performed as recommended by the experimental guidelines of the European Community and performed in accordance with the NIH Guide for the Care and Use of Laboratory Animals (1996). Three adult male C57BL/6j mice were obtained from Charles River (L'Arbresle, France). After lethal sodium pentobarbital intraperitoneal injection, testicles were removed by laparotomy. Confocal imaging was realized within $1 \mathrm{~h}$ after extraction on the left testis using an upright TCS SP2 microscope (Leica Microsystems, Wetzlar, Germany) equipped with a Plan-Apochromat $\times 20$ waterimmersion objective lens. Two-photon excitation was provided by a mode-locked Titanium-Sapphire laser system (Coherent Inc., Santa Clara, CA, USA) tuned to $800 \mathrm{~nm}$. Imaging was processed by applying the water-immersion objective directly on the connective tunica albuginea, which remained intact through the experiment. Fluorescence emitted by the tissues was collected in a range of 700-1000 $\mathrm{nm}$ on the confocal system photomultiplier.

The contralateral testis was fixed right after killing in Bouin's fixative, and histologically processed for staining using Masson's trichrome technique and microphotography. Tubule diameter quantifications were done on a total of 32 tubules (half for each

ILaboratoire d'Histologie-Cytologie, Faculté de Médecine secteur Timone, Université de la Méditerranée, Marseille 13385, France; ${ }^{2}$ Centre d'Assistance Médicale à la Procréation, AP-HM Conception, Marseille 13385, France; ${ }^{3}$ CNRS - IBDML - UMR6216, Service Imagerie - Case 907, Faculté des Sciences de Luminy, Marseille 13288, France and ${ }^{4}$ Centre de Microscopie et Imagerie, IFR Jean Roche, Université de la Méditerranée, Marseille 13916, France Correspondence: Dr Vincent Achard (vincent.achard@mail.ap-hm.fr)

Received: 7 November 2010; Revised: 23 December 2010; Accepted: 16 January 2011; Published online: 11 April 2011 
microscopic technique) using NIH ImageJ software after calibration. Results are given as mean \pm s.e. Student's $t$-test was used for statistical comparison.

\section{RESULTS AND DISCUSSION}

Two-photon microscopy revealed the autofluorescence of several tubule outlines under the reticulated connective fibers of the tunica albuginea ('TA', Figure 1a and b). The diameters of the tubules showed no significant variation related to the microscopic technique used: $212.2 \pm 4.4 \mu \mathrm{m}$ using two-photon microscopy versus $180.0 \pm$ $4.8 \mu \mathrm{m}(P>0.05)$ using standard microscopy.

Autofluorescent spikes of moderate intensity were regularly distributed within each tubule (Figure 1a and b). On each laser slice, a spotlike appearance was observed near the center of the tubule versus a spiked organization towards the outline. This particular setting was even more evident on a Z-stack (picture series along the vertical axis) which shows the three-dimensional structure (Supplementary data). Leydig cells could be identified according to their shape, typical triangular organization and intertubular situation (Figure 1a: 'LC'). Standard microscopy showed the normal testicular parenchyma in the right testis (Figure 1c and d).

Greenhalgh et al. ${ }^{7}$ have proposed to use an immunofluorescence technique to detect the presence of spermatogenetic foci in the parenchyma and guide microdissection. In this study, immunofluorescent antibodies and crystal violet were microinjected directly in the parenchyma. TPLSM uses long wavelengths for excitation (infrared light penetrates living tissues with minimal scattering or absorption); therefore, the energy transmitted to the tissue is low compared to other fluorescence-based microscopy. ${ }^{5}$ Using two-photon excitation restricted the excitation to the confocal volume. König et al. ${ }^{6}$ have studied the photodamage caused by light sources at several wavelengths on sperm cells. They found that within the ranges we use in the present study, no viability or motility loss could be evidenced, which was not the case for lower wavelengths.

Using two-photon microscopy we were able to identify autofluorescence within the testis. The collagen fibers of the tunica albuginea could easily be identified, along with the tubules outlines. Although not significant (probably because of the low number of tubules measured), the decrease in tubule diameter in fixed specimen is likely to be related to dehydratation during tissue processing for paraffin embedding. ${ }^{8}$ We detected autofluorescent structures within the seminiferous tubules that were globally linear, perpendicular to the seminiferous tubules basal membranes and seemed to occupy most of the height of the seminiferous epithelium, although this has to be confirmed. Autofluorescence is a common feature of fresh tissues, whose main intracellular sources are $\mathrm{NAD}(\mathrm{P}) \mathrm{H}$, flavins, retinol, tryptophan and lipofuscins. ${ }^{4,9}$ Most of these molecules are involved in the generation of ROS that immature spermatozoa can produce at variable levels. ${ }^{10}$ Lipofuscins are thought to accumulate mainly in post-mitotic cells; ${ }^{9}$ therefore, Sertoli cells are likely to be the autofluorescent spikes observed within the seminiferous tubules, which would correlate with their particular layout within the seminiferous epithelium. It has been shown that the number of Sertoli cells was correlated with sperm production. ${ }^{11-13}$ Peritubular cells are likely to be the strong autofluorescent tubules outlines. Importantly, a limitation in the current state of TPLSM development is the depth of observation ranging from 350 to $500 \mu \mathrm{m}$ which restricts the depth of observation to the region of the parenchyma under the tunica albuginea.
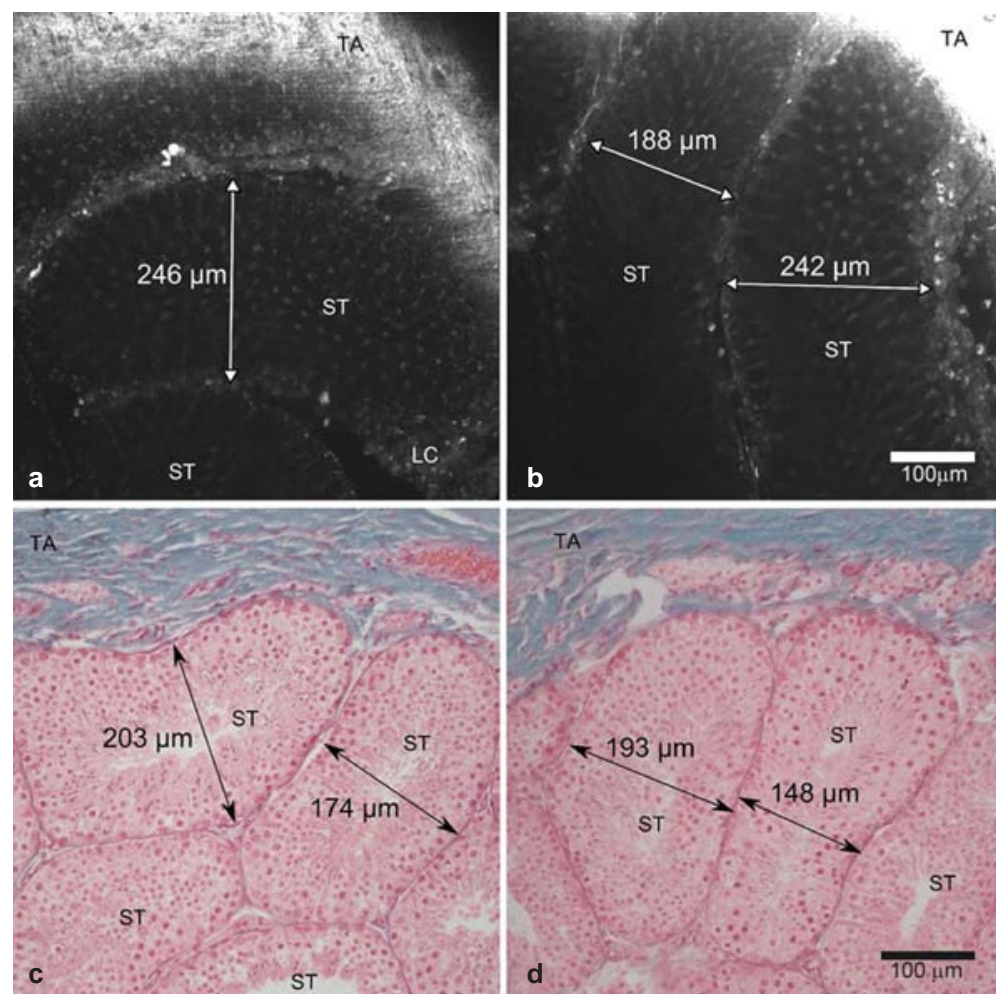

Figure 1 Photomicrographs of both testes obtained from the same animal. (a, b) Images obtained by live two-photon confocal scanning microscopy on a fresh whole testis through the TA without tissue preparation or staining, $1 \mathrm{~h}$ after extraction. (c, d) Images obtained from histological fields observed after classical histological processing of the other testis (fixation, paraffin embedding and trichrome counterstaining) and observation with a regular microscope. Lengths are given in micrometers $(\mu \mathrm{m})$. ST, seminiferous tubules; TA, tunica albuginea. 
In conclusion, using TPLSM we were able to observe seminiferous tubules and measure their diameters through the tunica albuginea on a fresh mouse testis without any fixation or drug administration. Elements within the tubules could also be evidenced and further experiments are needed to identify them.

Note: Supplementary information is available on the Asian Journal of Anrology website (http://www.nature.com/aja/).

\section{AUTHOR CONTRIBUTIONS}

VA conceived and designed the research and analysed data. PW and MPB performed TPLSM imaging. GM processed the samples histology.

\section{COMPETING FINANCIAL INTERESTS}

This work did not benefit from any financial aid, grant or equipment.

\section{ACKNOWLEDGMENTS}

We thank Dr Ilse Scroyen and Dr Delphine Bastelica for their help with animal manipulation.

1 Ramasamy R, Lin K, Gosden LV, Rosenwaks Z, Palermo GD et al. High serum FSH levels in men with nonobstructive azoospermia does not affect success of microdissection testicular sperm extraction. Fertil Steril 2009; 92: 590-3.
2 Ramasamy R, Yagan N, Schlegel PN. Structural and functional changes to the testis after conventional versus microdissection testicular sperm extraction. Urology 2005; 65: 1190-4.

3 Svoboda K, Yasuda R. Principles of two-photon excitation microscopy and its applications to neuroscience. Neuron 2006; 50: 823-39.

4 Zipfel WR, Williams RM, Christie R, Nikitin AY, Hyman BT et al. Live tissue intrinsic emission microscopy using multiphoton-excited native fluorescence and second harmonic generation. Proc Natl Acad Sci USA 2003; 100: 7075-80.

5 Cahalan MD, Parker I, Wei SH, Miller MJ. Two-photon tissue imaging: seeing the immune system in a fresh light. Nat Rev Immunol 2002; 2: 872-80.

6 König K, Tadir Y, Patrizio P, Berns MW, Tromberg BJ. Effects of ultraviolet exposure and near infrared laser tweezers on human spermatozoa. Hum Reprod 1996; 11: 2162-4.

7 Greenhalgh JR, Griffith TS, Wald M. The use of immunofluorescence in microdissection testicular sperm extraction. J Androl 2009; 30: 548-51.

8 Weiss L, Greep RO. Histology. New York: McGraw-Hill Publishers; 1977. p13.

9 Billinton N, Knight AW. Seeing the wood through the trees: a review of techniques for distinguishing green fluorescent protein from endogenous autofluorescence. Anal Biochem 2001; 291: 175-97.

10 Liu Z, Lin H, Ye S, Liu QY, Meng Z et al. Remarkably high activities of testicular cytochrome $\mathrm{c}$ in destroying reactive oxygen species and in triggering apoptosis. Proc Natl Acad Sci USA 2006; 103: 8965-70.

11 Berndtson WE, Igboeli G, Pickett BW. Relationship of absolute numbers of Sertoli cells to testicular size and spermatogenesis in young beef bulls. J Anim Sci 1987; 64: 241-6.

12 Orth JM, Gunsalus GL, Lamperti AA. Evidence from Sertoli cell-depleted rats indicates that spermatid number in adults depends on numbers of Sertoli cells produced during perinatal development. Endocrinology 1988; 122: 787-94.

13 Johnson L, Carter GK, Varner DD, Taylor TS, Blanchard TL et al. The relationship of daily sperm production with number of Sertoli cells and testicular size in adult horses: role of primitive spermatogonia. J Reprod Fertil 1994; 100: 315-21. 